\title{
Ethanol as Dentin Pretreatment on the Bonding Performance of a Two-Step Etch-and-Rinse Adhesive: An In Vivo Study
}

\author{
Francisco Cláudio Fernandes Alves Silva ${ }^{1}$ Victor Pinheiro Feitosa ${ }^{2}$ Vicente de Paulo Aragão Saboia ${ }^{2}$ \\ ${ }^{1}$ Department of Dentistry, Christus University Center, Fortaleza, \\ Ceará, Brazil \\ 2Department of Restorative Dentistry, Faculty of Pharmacy,

\begin{abstract}
Address for correspondence Francisco Cláudio Fernandes Alves Silva, PhD, R. Paula Ney, 599 Ap. 1401 - Aldeota Fortaleza-CE (Brazil) CEP 60140-200 (e-mail: claudiofernandes@me.com).
\end{abstract} Dentistry and Nursing, Federal University of Ceará, Fortaleza, Ceará, Brazil

Eur J Dent 2019;13:137-142

\begin{abstract}
Objective The aim of this study was to evaluate the use of 70 vol.\% ethanol as a dentin pretreatment on the bond strength (BS) of a two-step etch-and-rinse adhesive. Materials and Methods Resin composite Class I restorations were clinically bonded to acid-etched dentin of human sound third molars using Adper Single Bond 2 (SB, 3M ESPE) and randomly divided into two major groups: dentin saturated with water (control) or 70 vol.\% ethanol (ethanol). The teeth were divided into two subgroups: immediately extracted and tested after 24 hours and extraction after 18 months. Bonded teeth (Adper SB 2) were cut into resin-dentin sticks that were tested by microtensile $B S$, and the failure mode was thereafter evaluated.

Statistical Analysis Data were statistically analyzed using two-way analysis of variance and Holm-Sidak post hoc test $(\alpha=0.05)$. Additional bonded resin-dentin slabs from each group were examined under light microscopy (LM) using the Masson's trichrome staining technique.

Results The lowest BS was obtained by ethanol pretreated dentin after aging, while other groups presented similar BS. The LM analysis showed the presence of resin-sparse collagen fibrils in groups examined immediately ( 24 hours) and the presence of several

Keywords

- bond strength

- ethanol wet bonding

- hybrid layer gaps due to collagen degradation at the interfaces of ethanol pretreated aged specimens (18 months).

Conclusion The use of 70 vol.\% ethanol in dentin before the application of a two-step etch-and-rinse adhesive should be avoided once it resulted in a significant drop on the BS.
\end{abstract}

\section{Introduction}

The current concept of adhesion to dentin relies on the infiltration of monomers from the adhesive resin within the etched dentin tissue (collagen mesh), to allow the formation of the so-called hybrid layer. ${ }^{1}$ The hybrid layer is considered the foremost factor to achieve high bond strength (BS) between the restorative composite and the dentin., ${ }^{2,3}$ During this process, water is responsible for maintaining the collagen fibrils expanded that allows resin penetration and formation of hybrid layer after the subsequent polymerization of the resin. ${ }^{4,5}$ Although water has been important in this bonding mechanism, especially using etch-and-rinse adhesives, it is also associated with the degradation of the resin-dentin bonds. ${ }^{2,5-7}$ To simplify the application of dentin adhesives, the two-step etch-and-rinse and one-step self-etch adhesives were developed. However, they are intrinsically more hydrophilic than the multistep versions. In fact, such hydrophilic polymers result in significant water sorption, ${ }^{8,9}$ which also cause the decrease in mechanical properties. In addition, acid etching exposes and activates matrix metalloproteinases in the dentin that is able to accelerate the collagen degradation promoted by water., ${ }^{3,10,11}$ The residual water entrapped surrounding the collagen fibrils may impair the diffusion of 
more hydrophobic monomers, ${ }^{12-14}$ potentially contributing to a rapid resin-dentin interfacial degradation..$^{15,16}$ Thus, the residual water must be removed as much as possible to attain an optimal resin infiltration. ${ }^{17}$ Solvents such as acetone and ethanol are added to the adhesive resin blend to decrease its viscosity as well as to facilitate the evaporation of water from the interfibrillar spaces, thereby contributing to the formation of the hybrid layer. ${ }^{5}$ Certainly, the complete removal of this water is unattainable in user-friendly protocols.

In a clinical attempt to dehydrate the exposed collagen fibrils, a simplified dehydration protocol applying 100 vol.\% ethanol three times for 1 minute before the adhesive application was tested. ${ }^{18}$ Although this technique seems promising, it is still time-consuming and ethanol applied at this concentration may not be entirely effective due to its fast evaporation. ${ }^{19}$

The use of 70 vol.\% ethanol as a dentin pretreatment could be useful to reduce the overall residual water content to improve the more hydrophobic monomers infiltration ${ }^{18}$ and potentially to attain antimicrobial properties..$^{20}$

Therefore, this study aimed to evaluate clinically the effect of such dentin pretreatment adjunctively used with a twostep etch-and-rinse adhesive on the dentin BS and interfacial morphology. The null hypothesis to be tested was that 70 vol.\% ethanol dentin pretreatment does not interfere on the bonding performance of a two-step etch-and-rinse adhesive.

\section{Materials and Methods}

This research protocol was approved by the appropriate Institutional Research Ethics Committee (Process no. 108/10). Eight volunteers of both genders, with ages from 18 to 30 years, who presented all four sound third molars, erupted, in function, and orthodontically scheduled for extraction were selected for the study. After all, patients had signed informed consent form; the clinical procedures were performed.

\section{Bonding Procedures}

From the same patient, four teeth were randomly assigned to receive a restorative adhesive procedure. After acid etching, 70 vol.\% ethanol was applied in two teeth (experimental group), and in the control group (two teeth), ethanol was not applied. From each treatment, one tooth was extracted immediately ( 24 hours) and another tooth extracted at 18 months. The same clinical procedures were performed for all teeth: local anesthesia using anesthetics without vasoconstrictor (MEPISV 3\%-DFL, Rio de Janeiro, Brazil), rubber dam isolation, and Class I cavity preparation for composite resin, with continuous enamel cavosurface margins using diamond burs \#3131 (KG Sorensen, São Paulo, Brazil) underabundant water cooling. To obtain standardization, the depth of the cavities was controlled using the half-length of the diamond burs as a reference $(4 \mathrm{~mm})$ in the central sulcus, following the long axis of the teeth and the intercuspal distance controlled the width. The teeth were radiographically examined prior cavities preparation to avoid the use of teeth with morphological defects or caries. A single trained operator performed all the restorative procedures. Cavities were etched with 35\% phosphoric acid (Scotchbond Etchant; 3M-ESPE, St. Paul, Minnesota, United States) for 15 seconds and thoroughly rinsed with water for 15 seconds. The excess of water was removed with absorbent papers (Mellita; São Paulo, Brazil) according to the etch-and-rinse technique. Afterward, the experimental groups were saturated with 70 vol.\% ethanol for 20 seconds. In all groups, the two-step etch-andrinse adhesive Adper Single Bond (SB) 2 (3M/ESPE, St. Paul, Minnesota, United States) was applied to the cavity walls and light activated with a halogen lamp for 10 seconds (Optilux 500; Kerr, Danbury, Connecticut, United States) at $600 \mathrm{~mW} / \mathrm{cm}^{2}$, as periodically controlled by a radiometer (Demetron; Kerr). The buildups were constructed with a resin composite shade A2 (Filtek Z350 XT; 3M/ESPE, St. Paul, Minnesota, United States) in $1 \mathrm{~mm}$-thick increment; light-activated for 20 seconds each. The commercial brand, components, mode of application, and manufacturers of the materials used in the study are presented in - Table 1 .

The volunteers were informed about the oral hygiene and teeth were periodically followed up clinically and radiographically.

\section{Microtensile Bond Strength}

Teeth were collected, using the minimal traumatic technique of extraction to avoid damages to dental structures. Bonded teeth were cut into beam-shaped specimens with a cross-sectional area of $\sim 0.8 \mathrm{~mm}^{2}$ using a slow-speed watercooled diamond saw (Isomet 1000, Buehler. Lake Bluff, Illinois, United States). Each beam was measured with a digital caliper (Absolute Digimatic, Mitutoyo, Tokyo, Japan), fixed

Table 1 Materials used in the study

\begin{tabular}{|l|l|l|l|}
\hline Commercial brand & Main components & Mode of application & Manufacturer \\
\hline Adper Single Bond 2 & $\begin{array}{l}\text { Ethanol, Bis-GMA, HEMA, } \\
\text { GDMA, polycarboxylic acid } \\
\text { copolymer, UDMA, water, CQ, } \\
\text { EDMAB, DP1FHP }\end{array}$ & $\begin{array}{l}\text { Apply one layer of adhesive, } \\
\text { wait for 20 s, air stream for } 5 \\
\text { s, and polymerize for } 10 \mathrm{~s}\end{array}$ & $3 \mathrm{M} / \mathrm{ESPE}$ \\
\hline Scotchbond Etchant & $\begin{array}{l}35 \% \text { phosphoric acid, water, } \\
\text { silica }\end{array}$ & $\begin{array}{l}\text { Apply a layer for } 15 \mathrm{~s} \text { and wash } \\
\text { for } 15 \mathrm{~s}\end{array}$ & $3 \mathrm{M} / \mathrm{ESPE}$ \\
\hline Filtek Z350 XT & $\begin{array}{l}\text { Bis-GMA, UDMA, Bis-EMA, } \\
\text { TEGDMA resins, zirconium, } \\
\text { silica }\end{array}$ & $\begin{array}{l}\text { Apply increments of } 2 \mathrm{~mm} \\
\text { and polymerize for } 20 \mathrm{~s}\end{array}$ & $3 \mathrm{M} / \mathrm{ESPE}$ \\
\hline
\end{tabular}


to a test apparatus (Bencor Multi-T Device-Danville Engineering, San Ramon, California, United States) using cyanoacrylate glue (Zapit, Dental Ventures of America, Corona, California, United States), submitted to microtensile BS $(\mu \mathrm{TBS})$ test in a universal testing machine (Instron 4411, Canton, Ohio, United States), and stressed until failure with a tensile force at a speed of $1 \mathrm{~mm} / \mathrm{min}$; data were collected in MPa. The data (MPa) attained from the beams of the same resin-bonded tooth were averaged and the mean BS was used as 1 unit for statistical analysis. Immediately after testing, the debonded beams were dried and stored at room temperature until analysis of the fracture pattern using a stereomicroscope (Stemi 2000-C, Carl Zeiss Jena GmbH, Germany) at $\times 50$ magnification. Failure mode was classified as cohesive failure in dentin, cohesive in resin, adhesive $(A)$, or mixed failure $(\mathrm{M})$.

\section{Statistical Analysis}

The Statistical Package for the Social Sciences version 17.0 (SPSS, Chicago, Illinois, United States) software was used to perform the statistical analysis. A two-way analysis of variance (ANOVA) was applied to the $\mu$ TBS data to analyze the factors "dentin pretreatment" (water versus ethanol) and "aging" (24 hours vs. 18 months), complemented by Holm-Sidak multiple comparison post hoc test $(p<0.05)$. The significance level was set at $\alpha=0.05$ and statistical unit was tooth $(n=4)$. Premature failures were noted, but not included in the data analysis.

\section{Light Microscopy-Masson's Trichrome}

One tooth from each group was sectioned in only one direction to obtain 1-mm thick dentin-resin slabs, which were fixed on a glass holder with cyanoacrylate glue (Super Bonder Flex Gel-Henkel Ltd., Düsseldorf, Germany) and polished with $\mathrm{SiC}$ papers on increasing fine grits $(800,1000,1200$, and 2500$)$ under running water (Buehler, Lake Bluff, Illinois, United States), reducing the slabs to $\sim 150 \mu \mathrm{m}$ in thickness. After polishing, the specimens were treated with Masson's trichrome staining technique as previously described. ${ }^{21}$ This staining technique has high affinity for cationic elements normally found in mineralized type I collagen, resulting in the blue color. The acid etching of dentin causes the removal of these cationic elements and exposes collagen fibers showing a red pigmentation. These exposed collagen fibrils showed in the light microscopy (LM) images, represented by a thin red-colored layer at the $\mathrm{HL}$, is called "red zone." Using this microscopic technique, lower incidence of red zones at the interface indicates less denuded collagen fibrils. ${ }^{11,22}$ The composite resin usually stains in beige color. After all staining procedures, the specimens were covered with a glass coverslip and analyzed under LM at $\times 400$ magnification (Olympus BH-2, Tokyo, Japan). The evaluation of Masson's trichrome was performed qualitatively.

\section{Results}

\section{Microtensile Bond Strength}

Two-way ANOVA test showed there is a statistically significant interaction between treatment and aging $(p=0.008)$. Holm-Sidak multiple comparison post hoc test showed that the BS was not affected by ethanol pretreatment 24 hours after restoration $(p=0.430)$. After 18 months, no BS reduction was observed in water-saturated dentin $(p=0.096)$, but a significant drop in BS was attained using ethanol pretreatment $(p<0.001)$, with a statistically significant difference between the two dentin treatments after aging $(p<0.001)$. Mixed failures (M) were the most common fracture pattern observed in all groups. In the ethanol groups, adhesive failures (A) were three times more frequent after 18 months than in 24 hours. In the control groups, this failure pattern remained predominant. Mean BS, standard deviations, and failure mode distribution are summarized in - Table 2.

\section{Light Microscopy: Masson's Trichrome}

LM showed resin-sparse collagen fibrils within the resin-dentin interfaces in immediate groups, demonstrated by the red zones ( $\boldsymbol{- F i g s .} \mathbf{1 A}, \mathbf{C}$ ), with greater intensity in the group treated with ethanol. For the aged groups (18 months), the group treated with water showed some isolated, discrete, and less colored red zones at the bonded interface and white zone suggesting the absence of either polymers or collagen

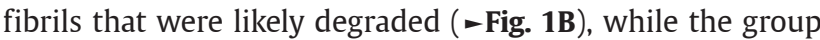
treated with ethanol showed no red zone but white zone ( - Fig. 1D).

Table 2 Bond strength and distribution of failure modes

\begin{tabular}{|c|c|c|c|c|c|c|c|c|c|c|}
\hline & $\mu \mathrm{TB}$ & $(\mathrm{APA})^{\mathrm{a}}$ & & & & ilure & les ( & & & \\
\hline & & & & & ours & & & & & \\
\hline & 24 hours & 18 months & $C D$ & CR & $M$ & $A$ & $C D$ & CR & $M$ & $A$ \\
\hline Control & $31.7(3.9)^{\mathrm{A}}$ & $31.5(3.8)^{\mathrm{A}}$ & 9 & 18 & 61 & 12 & 9 & 12 & 65 & 14 \\
\hline Ethanol & $30.3(4.3)^{A}$ & $21.9(3.2)^{\mathrm{B}}$ & 4 & 6 & 83 & 7 & 5 & 6 & 68 & 21 \\
\hline
\end{tabular}

${ }^{a}$ Bond strength values are means (SD). Different superscripts indicate statistically significant difference $(p<0.05)$.

bFailure modes pattern: $C D$, cohesive failure in dentin; $C R$, cohesive failure in resin; $M$, mixed failure; $A$, adhesive failure; $\mu$ TBS, microtensile bond strength, SD, standard deviations).

Note: Different subscript letters indicate statistically significant difference $(p<0.05)$. 


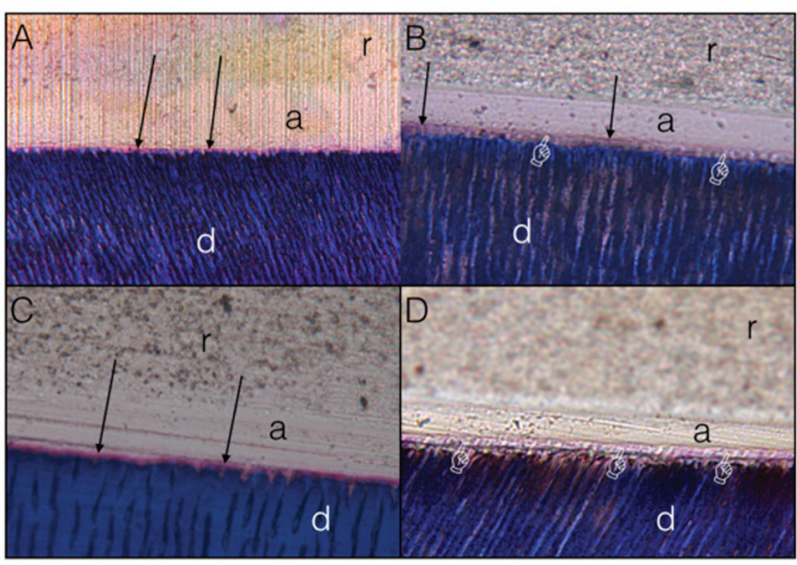

Fig. 1 Light micrographs Masson's trichrome of resin-dentin interface: (A) Control immediate; (B) control aged; (C) ethanol immediate; (D) ethanol aged. a, adhesive; $d$, dentin; $r$, resin. Arrows-red zone, indicating the presence of denuded collagen fibrils; Pointerswhite zone indicating the absence of both polymer or exposed collagen fibril, suggesting degradation of resin-dentin interface

\section{Discussion}

Based on the outcomes aforementioned, the null hypothesis was rejected once ethanol pretreated dentin showed a significant decrease in the BS after 18 months in clinical function.

The residual water in the dentin matrix and the hydrophilic domains of contemporary dental adhesives make the hybrid layer behave as a semipermeable membrane, which permits water permeation through the bonded interface even after polymerization. ${ }^{3,23}$ By dentin acid etching, the etchant dissolves interfibrillar apatite crystallites and exposes the collagen, creating spaces between and inside the collagen fibrils. ${ }^{16}$ Several authors have reported that the presence of a hydrogel composed of proteoglycans in these spaces. ${ }^{24-28}$ Studies affirm that the presence of this hydrogel may interfere with monomer infiltration during bonding, and the removal of water from these spaces results in hydrogel collapse. ${ }^{8,29,30}$ The solvated resins of contemporary twostep etch-and-rinse adhesives do not remove all the residual water from the interfibrillar spaces, ${ }^{2,31,32}$ leaving small amount of water into the partially demineralized dentin that may hinder the hydrophobic comonomer blend to optimally coat the exposed collagen. ${ }^{31,33}$ Based on the solubility parameters theory of Hansen, ${ }^{34-36}$ ethanol is miscible with both hydrophobic monomers and water, which makes this substance an appropriate alternative to facilitate the penetration of hydrophobic monomers into a water-wet substrate. ${ }^{29}$ The so-called ethanol-wet-bonding technique relies on filling spaces between the fibrils with ethanol, ${ }^{37}$ thereby replacing all water in the partially demineralized dentin by ethanol. ${ }^{38}$ It may permit hydrophobic comonomer blend to infiltrate the spaces along the etched substrate properly, providing less resin-sparse collagen, and consequently durable resin-dentin bonds. ${ }^{19,39,40}$

Experiments with different concentrations of ethanol have been applied in a simplified protocol, also using 70 vol.\% and 100 vol.\%. ${ }^{18,39-41}$ However, when a simplified protocol is applied using ethanol at high concentration (i.e., 100 vol.\%), the replacement of water may not be totally efficient to promote a completely saturated substrate, resulting in relatively low infiltration of hydrophobic monomers. ${ }^{11,40,42} \mathrm{~A}$ suitable explanation for this occurrence could be the high vapor pressure of $100 \mathrm{vol} . \%$ ethanol, which is almost three times higher than that of water. ${ }^{37}$ In spite of 70 vol.\% ethanol has a relatively high volatility, the presence of water in its composition decreases its evaporation time and vapor pressure in comparison with 100 vol.\%, thereby facilitating the exchange of water and the maintenance of interfibrillar spaces. ${ }^{18}$ Yet, water facilitates the passage of ethanol through the bacterial cell wall that attains its well-known antibacterial properties. ${ }^{20}$ Moreover, 70 vol.\% ethanol dentin pretreatment showed a significant increase on the initial BS of Adper SB 2 (unpublished observations, 2013). Therefore, 70 vol.\% ethanol could be a feasible alternative as a dentin pretreatment as used in this study.

In spite of using ethanol, our study did not apply the ethanol-wet-bonding technique, but rather a simple protocol (70 vol.\% ethanol for 20 second of dentin pretreatment) to advocate the well-known characteristics of ethanol. The immediate results were promising, showing ethanol with similar BS of the control group. Nevertheless, the amount of red zone (resin-sparse collagen) was lower in the water-treated group ( $\boldsymbol{- F i g . 1 A}$ ). However, when the ethanol pretreatment was used in such an adverse situation as clinical function aging (18 months), the results were discouraging once the BS strikingly decreased and the resin-dentin interface was severely degraded ( - Fig. 1D). The negative outcomes of ethanol pretreated aged group could be explained by the ineffectiveness of $70 \mathrm{vol}$ \% ethanol to remove all residual water from the interfibrillar spaces. One the contrary, the amount of water in its composition may have increased the moisture of the partially demineralized substrate, impairing an efficient coating of the exposed collagen fibrils by resin (represented by the increase in the red zones) ( - Fig. 1C) and jeopardizing the solvent evaporation.

Our findings are in accordance with those of Huang et a ${ }^{43}$ and de Barros et $\mathrm{al}^{44}$ who found a decrease on the BS and significant collagen degradation in specimens pretreated with 100 vol.\% ethanol and submitted to thermocycling or immersion in $\mathrm{NaOCl}$. Conversely, Hosaka et al, ${ }^{42}$ Pashley et a ${ }^{37}$ and Carvalho et al, ${ }^{45}$ showed a strong increase in BS using the ethanol-wet-bonding technique. Although Pashley's in vitro study $^{37}$ had been performed using a macromodel of hybrid layer, differently from an in vivo study, in which some variables are difficult to control such as thickness of the smear layer, cavity preparation, dentin moisture, and intrapulpal pressure. ${ }^{46,47}$ However, in a clinical situation, resin-dentin interfaces are only partially in contact with environmental fluids, since outer resin-bonded enamel has been shown to prevent water uptake. ${ }^{12,45,46}$ In such circumstances, these resin-dentin bonds may come in contact with fluids in vivo only through pulpal pressure through the dentinal tubules ${ }^{40}$ or by residual water in etched and rinsed dentin. ${ }^{45}$

White spaces found in the resin-dentin interfacial microscopies after aging, where there was the red zone staining ( - Figs. 1B and D), suggest the breakdown of resin-infiltrated 
dentin ${ }^{46}$ and/or collagenolytic degradation, which may be governed by host-derived factors, such as the action of endogenous collagenolytic enzymes on partially exposed collagen fibrils. ${ }^{15}$ Nevertheless, the ethanol pretreatment resulted in a significant decrease on BS in the long term that was not observed using water (control). Furthermore, the ethanol aged group (18 months) showed an increase in adhesive failures (-Table 2) when compared with ethanol immediate (24 hours), which is another indication of resin-dentin degradation. It may be suggested that the disappearance of resin-sparse collagen layer as aforementioned and the appearance of gaps in the hybrid layer may have contributed to achieving this lower BS (- Table 1). However, the correlation between the decrease on BS and these gaps at the resin-dentin interface is complex and still unclear as discussed in the previous publication. ${ }^{2}$

Finally, further research is required to explain the many possible reasons for the degradation promoted by 70 vol.\% ethanol dentin pretreatment.

\section{Conclusion}

Within the limitations of this in vivo study, the following conclusions may be drawn:

1. Dentin saturation with ethanol 70 vol\% before the application of a two-step etch-and-rinse adhesive does not afford any improvement on the initial BS.

2. Ethanol saturation of dentin with ethanol $70 \mathrm{vol} \%$ jeopardizes the long-term bonding depicting interfacial degradation of simplified etch-and-rinse adhesive, thereby suggesting it should not be applied clinically.

\section{Funding}

None.

\section{Conflict of Interest}

None declared.

\section{References}

1 Nakabayashi N, Kojima K, Masuhara E. The promotion of adhesion by the infiltration of monomers into tooth substrates. J Biomed Mater Res 1982;16(3):265-273

2 Breschi L, Mazzoni A, Ruggeri A. Cadenaro M, Di Lenarda R, De Stefano Dorigo E. Dental adhesion review: aging and stability of the bonded interface. Dent Mater 2008;24(1):90-101

3 Tay FR, Pashley DH. Water treeing-a potential mechanism for degradation of dentin adhesives. Am J Dent 2003;16(1):6-12

4 De Munck J, Van Meerbeek B, Yoshida Y, et al. Four-year water degradation of total-etch adhesives bonded to dentin. J Dent Res 2003;82(2):136-140

5 De Munck J, Van Landuyt K, Peumans M, et al. A critical review of the durability of adhesion to tooth tissue: methods and results. J Dent Res 2005;84(2):118-132

6 Carvalho RM, Manso AP, Geraldeli S, Tay FR, Pashley DH. Durability of bonds and clinical success of adhesive restorations. Dent Mater 2012;28(1):72-86

7 Hashimoto M, Ohno H, Kaga M, Endo K, Sano H, Oguchi H. In vivo degradation of resin-dentin bonds in humans over 1 to 3 years. J Dent Res 2000;79(6):1385-1391
8 Ito S, Hashimoto M, Wadgaonkar B, et al. Effects of resin hydrophilicity on water sorption and changes in modulus of elasticity. Biomaterials 2005;26(33):6449-6459

9 Malacarne J, Carvalho RM, de Goes MF, et al. Water sorption/solubility of dental adhesive resins. Dent Mater 2006;22(10):973-980

10 Loguercio AD, Uceda-Gomez N, Carrilho MR. Reis A. Influence of specimen size and regional variation on long-term resin-dentin bond strength. Dent Mater 2005;21(3):224-231

11 Nishitani Y, Yoshiyama M, Donnelly AM, et al. Effects of resin hydrophilicity on dentin bond strength. J Dent Res 2006;85(11):1016-1021

12 Reis AF, Giannini M, Pereira PN. Effects of a peripheral enamel bond on the long-term effectiveness of dentin bonding agents exposed to water in vitro. J Biomed Mater Res B Appl Biomater 2008;85(1):10-17

13 Spencer P, Wang Y. Adhesive phase separation at the dentin interface under wet bonding conditions. J Biomed Mater Res 2002;62(3):447-456

14 Tay FR, Pashley DH, Kapur RR, et al. Bonding BisGMA to dentin-a proof of concept for hydrophobic dentin bonding. J Dent Res 2007;86(11):1034-1039

15 Carrilho MR, Geraldeli S, Tay F, et al. In vivo preservation of the hybrid layer by chlorhexidine. J Dent Res 2007;86(6):529-533

16 Van Meerbeek B, De Munck J, Yoshida Y, et al. Buonocore memorial lecture. Adhesion to enamel and dentin: current status and future challenges. Oper Dent 2003;28(3):215-235

17 Vaidyanathan TK, Vaidyanathan J. Recent advances in the theory and mechanism of adhesive resin bonding to dentin: a critical review. J Biomed Mater Res B Appl Biomater 2009;88(2):558-578

18 Li F, Liu XY, Zhang L, Kang JJ, Chen JH. Ethanol-wet bonding technique may enhance the bonding performance of contemporary etch-and-rinse dental adhesives. J Adhes Dent 2012;14(2):113-120

19 Sadek FT, Castellan CS, Braga RR, et al. One-year stability of resin-dentin bonds created with a hydrophobic ethanol-wet bonding technique. Dent Mater 2010;26(4):380-386

20 Chambers ST, Peddie B, Pithie A. Ethanol disinfection of plastic-adherent micro-organisms. J Hosp Infect 2006;63(2):193-196

21 Garcia Del Moral R, ed. Colorations for collagen and elastic fibers of connective tissue, colorations for amyloid substance. In: Laboratory of Pathological Anatomy. Ch. 10 Madrid: McGraw-Hill/Interamerican of Sapin; 1993:175-178

22 Carvalho RM, Mendonça JS, Santiago SL, et al. Effects of HEMA/ solvent combinations on bond strength to dentin. J Dent Res 2003;82(8):597-601

23 Hashimoto M, Ito S, Tay FR, et al. Fluid movement across the resin-dentin interface during and after bonding. J Dent Res 2004;83(11):843-848

24 Embery G, Hall R, Waddington R, Septier D, Goldberg M. Proteoglycans in dentinogenesis. Crit Rev Oral Biol Med 2001;12(4):331-349

25 Goldberg M, Takagi M. Dentine proteoglycans: composition, ultrastructure and functions. Histochem J 1993;25(11):781-806

26 Oyarzún A, Rathkamp H, Dreyer E. Immunohistochemical and ultrastructural evaluation of the effects of phosphoric acid etching on dentin proteoglycans. Eur J Oral Sci 2000;108(6):546-554

27 Pereira PN, Bedran-de-Castro AK, Duarte WR. Yamauchi M. Removal of noncollagenous components affects dentin bonding. J Biomed Mater Res B Appl Biomater 2007;80(1):86-91

28 Toledano M, Osorio R, Perdigao J, Rosales JI, Thompson JY, Cabrerizo-Vilchez MA. Effect of acid etching and collagen removal on dentin wettability and roughness. J Biomed Mater Res 1999;47(2):198-203 
29 Bertassoni LE, Orgel JP, Antipova O, Swain MV. The dentin organic matrix - limitations of restorative dentistry hidden on the nanometer scale. Acta Biomater 2012;8(7):2419-2433

30 Cadenaro M, Breschi L, Rueggeberg FA, et al. Effect of adhesive hydrophilicity and curing time on the permeability of resins bonded to water vs. ethanol-saturated acid-etched dentin. Dent Mater 2009;25(1):39-47

31 Guo X, Spencer P, Wang Y, Ye Q, Yao X, Williams K. Effects of a solubility enhancer on penetration of hydrophobic component in model adhesives into wet demineralized dentin. Dent Mater 2007;23(12):1473-1481

32 Loguercio AD, Reis A, Ballester RY. Polymerization shrinkage: effects of constraint and filling technique in composite restorations. Dent Mater 2004;20(3):236-243

33 García-Godoy F, Tay FR, Pashley DH, Feilzer A, Tjäderhane L, Pashley EL. Degradation of resin-bonded human dentin after 3 years of storage. Am J Dent 2007;20(2):109-113

34 Asmussen E, Uno S. Solubility parameters, fractional polarities, and bond strengths of some intermediary resins used in dentin bonding. J Dent Res 1993;72(3):558-565

35 Barton AF, Handbook of Solubility Parameters and Other Cohesion Parameters. Boca Raton: CRC Press; 1991:69-156

36 Miller RG, Bowles CQ Chappelow CC, Eick JD. Application of solubility parameter theory to dentin-bonding systems and adhesive strength correlations. J Biomed Mater Res 1998;41(2):237-243

37 Pashley DH, Tay FR, Carvalho RM, et al. From dry bonding to water-wet bonding to ethanol-wet bonding. A review of the interactions between dentin matrix and solvated resins using a macromodel of the hybrid layer. Am J Dent 2007;20(1):7-20

38 Ayar MK. A review of ethanol wet-bonding: principles and techniques. Eur J Dent 2016;10(1):155-159
39 Osorio E, Toledano M, Aguilera FS, Tay FR, Osorio R. Ethanol wet-bonding technique sensitivity assessed by AFM. J Dent Res 2010;89(11):1264-1269

40 Pashley EL, Zhang Y, Lockwood PE, Rueggeberg FA, Pashley DH. Effects of HEMA on water evaporation from water-HEMA mixtures. Dent Mater 1998;14(1):6-10

41 Sadek FT, Mazzoni A, Breschi L, Tay FR, Braga RR. Six-month evaluation of adhesives interface created by a hydrophobic adhesive to acid-etched ethanol-wet bonded dentine with simplified dehydration protocols. J Dent 2010;38(4):276-283

42 Hosaka K, Nishitani Y, Tagami J, et al. Durability of resin-dentin bonds to water- vs. ethanol-saturated dentin. J Dent Res 2009;88(2):146-151

43 Huang X, Li L, Huang C, Du X. Effect of ethanol-wet bonding with hydrophobic adhesive on caries-affected dentine. Eur J Oral Sci 2011;119(4):310-315

44 de Barros L, Apolonio FM, Loguercio AD, de Saboia V. Resin-dentin bonds of etch-and-rinse adhesives to alcohol-saturated acid-etched dentin. J Adhes Dent 2013;15(4):333-340

45 Carvalho CA, Cantoro A, Mazzoni A, Goracci C, Breschi L, Ferrari M. Effect of ethanol application on post-luting to intraradicular dentine. Int Endod J 2009;42(2):129-135

46 Feitosa VP, Leme AA, Sauro S, et al. Hydrolytic degradation of the resin-dentine interface induced by the simulated pulpal pressure, direct and indirect water ageing. J Dent 2012;40(12):1134-1143

47 Saboia VP, Silva FC, Nato F, et al. Analysis of differential artificial ageing of the adhesive interface produced by a two-step etch-and-rinse adhesive. Eur J Oral Sci 2009;117(5):618-624 